\title{
VI. \\ Das Naturgefühl bei Platon.
}

Von

\author{
Dr. Willy Moog. \\ (Griesheim bei Dämstadt.)
}

Nicht plötzlich wie ein Blitz fuhr das Selbstbewußtsein in die Seele des Menschen, nicht mit einem einzelnen Moment gewann der Giist jene ungeheure Macht, sich über die Zufälligkeiten des äußeren Daseins zu erheben, sich selbst zum Objekt des Denkens zu nehmen und die Wissenschaft, speziell die Philosophie zu konstituieren, sondern erst allmählich haben sich aus der undifferenzierten Einheit des primitiven Lebens jene verschiedenen Reiche mit eigenen Gasetzen und Zwecken losgelöst, die in ihrer Vielgestaltigkeit das komplizierte $\mathrm{G}$ sbilde der Kultur repräsentieren. Aus mythischen und religiösen Anschauungen, wie sie die Epoche des sogenannten Naturzustandes charakterisieren, ist die Philosophie herausgewachsen, und gerade sie konnte nur schwer sich aus ihren ursprünglichen Voraussetzungen heraus zur Autonomie erheben, da sie mit ihren Problemen am tiefsten eindringen will in das Wesen des Menschen und der Welt und eine eigenartige Synthese von Subjektivem und Objektivem darstellt. Und noch längst, nachdem der Inhalt des Denkens eine Selbständigkeit und Eigenwertigkeit beansprucht, zeigt die äußere Form, in der sich dieser Inhalt ausdrückt, Reste überwundener Vorstellungen. So ist es zu erklären, daß sich bei den ersten griechischen Philosophen noch mannigfach mythische und poetische Elemente finden, daß sie, noch ungeschult, feste Begriffe zu formulieren, ihre Gadanken durch symbolische und metaphorische Beziehungen zu verdeutlichen suchen. Xenophanes, Parmenides, Empedokles bedienen sich noch der Form der gebundenen Rede, Heraklit schreibt eine Prosa, die von schwerverständlichen Bildern durchsetzt ist. Erst Aristoteles hat eine ausreichende Terminologie der Begriffe und damit den philosophischwissenschaftlichen Stil geschaffen. Bei seinem großen Lehrer Platon finden wir noch einmal eine Verbindung von Poesie und Philosophie, 
von Mythischem und Abstrakt-gedanklichem, eine Verbindung, die nicht so sehr auf Rechnung der zeitlichen Bedingtheit und Beschränkung zu setzen ist, sondern in der innersten Natur des Denkers begriindet liegt. Weil seine Seele reich ist an bunten Formen und Gastalten, weil sie ïberquillt von. Weisheit und Schönheit, weil sie künstlerisch schaffensfroh und schwer von Problemen ist, darum wird er so oft zum Dichter, wenn er philosophiert. Platon versteht es einerseits, das $\mathrm{G}$ sistige rein herauszuarbeiten, andererseits aber besitzt er auch Sinn für die konkrete. Wirklichkeit und Anschaulichkeit der äußeren Welt. So gewinnt er ein besonderes Verhältnis von Subjektivem und Objektivem, so tritt sein Ich auch zur "Natur" im speziellen Sinn in jene innige Beziehung, deren gefühlsmäßigen Reflex wir als Naturgefühl bezeichnen.

In der eigenartigen künstlerischen Form der platonischen Dialone ist es bedingt, $\mathrm{da}$ s sich die der philosophischen Spekulation fremd'n Elemente in verschiedener Weise geltend machen können. Äußerungen von Gefühl, Empfindung, Phantasie, die eine Relation des Ich zur Natur bezeichnen, können auftreten in poetischen Bildern, Gleichnissen, Metaphern und Beispielen, dann in Schilderungen und Beschrribungen, die meist der Ausmalung der Szenerie dienen und oft auf bloße Namen und Ortsangaben reduziert sind, schließlich in den Mythen. wo das Naturgefühl eine besondere Verbindung mit gedanklichen und phantasiemäßigen Konstruktionen eingehen kann.

Wenn ich im folgenden mit Zahlenmaterial operiere, so betone ich, daß dieses keinen Anspruch auf Vollständigkeit machen kann, sondern ich mußte mich darauf beschränken, das Wesentliche herauszuheben und etwa triviale Bilder und Metaphern, die für die Persönlichkeit des Schriftstellers nicht von Bedeutung sind, unberücksichtigt lassen.

Ich unterscheide unter den Mitteln, die zur Veranschaulichung und Verdeutlichung von Gedanken dienen oder eine Art von Gafühlsanteil bezeichnen: mehr oder weniger ausgeführte Gleichnisse, die meist eine poetische Färbung besitzen, kürzere Vergleiche und einzelne metaphorische Worte, dann bloße Beispiele, die gewöhnlich nur eine gedankliche Beziehung auf analoge Fälle oder die rein sachliche Anwendung eines allgemeinen Gedankens auf einen speziellen Fall enthalten. Natürlich drückt die Unterscheidung nur eine graduelle Abstufung aus, wobei mancherlei Utbergänge und Schwankungen stattfinden können. 
Am stärksten sprechen sich die Gefühlsmomente in den G l e i c h$n$ is s e $n$ aus. Doch läßt sich kaum eine direkte Gafühlsäußerung aufweisen, sondern das Maßgebende bleibt die Verbindung mit der Reflexion. Der Sinn für das konkrete Leben zeigt sich darin, daß die meisten Gleichnisse aus dem Gebiet menschlicher Tätigkeiten oder Zustände entnommen sind. Gleichnisse, die sich rein auf die Natur und besonders die unorganische beziehen, sind dagegen ziemlich selten. Namentlich handwerksmäßige oder gewerbliche physische Beschäftigungen werden gern zum Vergleich herangezogen. So heißt es

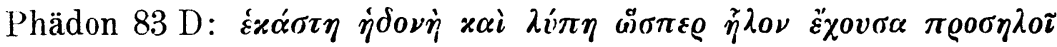

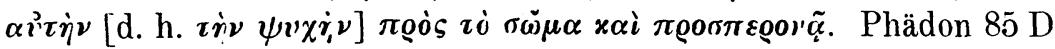
steht ein schon ziemlich unindividuelles Gleichnis von der Schiffahrt:

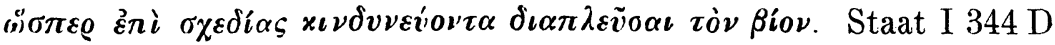

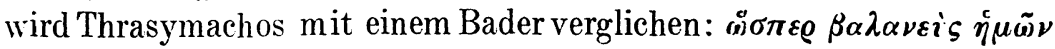

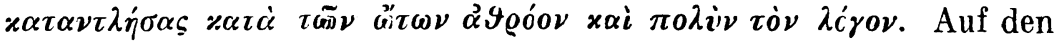

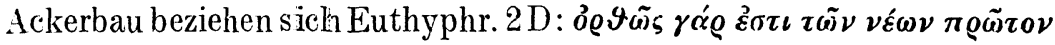

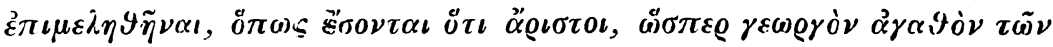

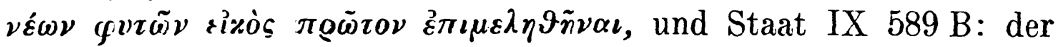

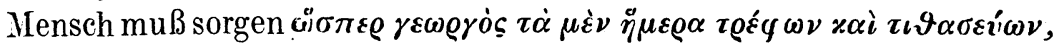

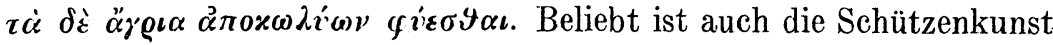
als Vergleichsobjekt: Theät. $194 \mathrm{~A}$ der Mensch kann in der falschen

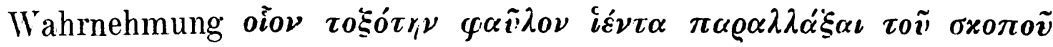

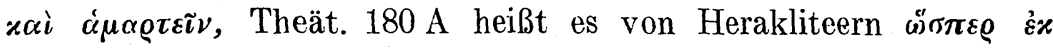

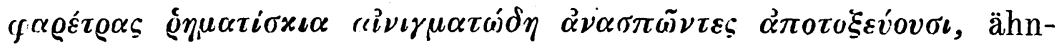

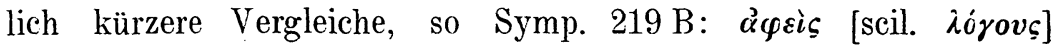

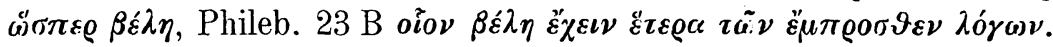
Auch mit sportlichen Fertigkeiten werden die Reden verglichen:

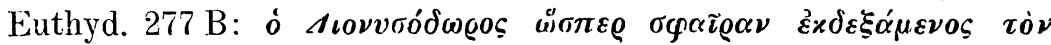

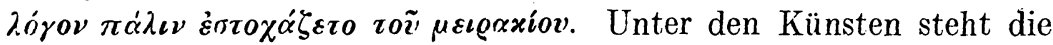

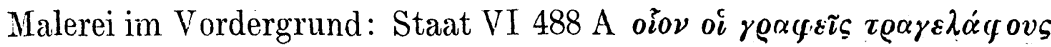

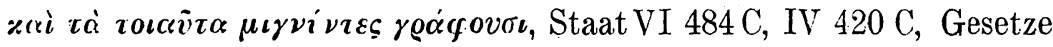
VI $769 \mathrm{~B}$ u. a. Menschliche Empfindungen, Reaktionen und Betätigungen des Organismus werden beobachtet und gleichnisweise verwandt, so das Zahnen Phädr. $251 \mathrm{C}$, das Gähnen Charmid. $169 \mathrm{C}$. Auch seelische Erscheinungen werden herangezogen: so die Zerstreutheit Staat IV 432 D, der Zustand der Verzückung Kriton 54 D; dann besondere Eigenschaften wie die Naschhaftigkeit Staat I 354 B. Auch Spiel und Scherz werden erwähnt: Theät. 181 A Spiel 
in der Palästra, Staat VI 487 B Brettspiel, X 604 C Würfel, IV 436 D Kreisel, Theät. 146 A ein Kinderspiel; auf einen etwas groben Spaß weist Euthyd. 278 B. Auf Nahrungsmittel, Speise und Trank beziehen sich verschiedene Gleichnisse: Timäus $52 \mathrm{E}$ ist von Durchsieben des Getreides die Rede, Gesetze I 638 C von Käse, Theät. 144 B von der Milde des Öls; Gesetze VI $773 \mathrm{C}$ wird die $\pi$ ó $\iota \varsigma$ mit einem Mischkrug verglichen, Phileb. $61 \mathrm{C}$ wird die Lust gleichnisweise eine Quelle von

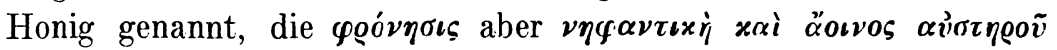

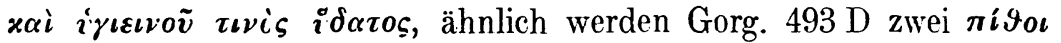
mit verschiedenem Inhalt eingeführt. Kleidung und Geräte, wie sie der Mensch braucht, werden mehrfach erwähnt. Der Staat wird mit einem Gewand verglichen Staat VIII 557 C, ähnlich Gesetze V 734 E. Die Wage dient zum Vergleich Staat VIII $550 \mathrm{E}$, eherne Becken werden herangezogen Protag. 329 A. Staat VII 519 B werden Bleikugeln ( $\left.\mu \circ \lambda_{v} \beta \delta \delta i \delta \varepsilon \varsigma\right)$ im Gleichnis gebraucht, ähnlich Gesetze I $644 \mathrm{E}$

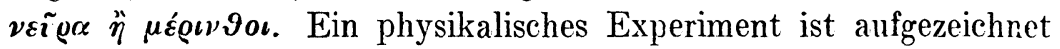
Sympos. 175 D. - Alle diese Gleichnisse sind direkt dem praktischen menschlichen Leben entnommen oder zeigen doch eine deutliche Beziehung zu ihm. Sie sind meist sehr anschaulich und offenbaren eine scharfe Beobachtungsgabe für Vorgänge des Physischen wie des Psychischen. Selbst da, wo sie phantasiemäßig konstruiert sind wie Gorg. $493 \mathrm{D}$ und keine unmittelbare Wiedergabe wirklicher Zustände sein können, enthalten sie doch in ihren Elementen eine konkrete Wahrscheinlichkeit. Von solchen Gleichnissen aus dem menschlichen Leben zähle ich 116 bei Platon, von solchen aus dem Tierleben 28 und von solchen, die auf die Natur im engeren Sinn weisen, 24. Unter den Tieren werden am häufigsten die Haustiere genannt, in erster Linie Pferd und Hund. Auch mit dem Leben der Bienen scheint Platon genau vertraut zu sein (cfr. Phädon 91 C, Politikos 293 D, Jon 534 B): Gleichnisse über Vögel finden sich drei in den Gesetzen (III $680 \mathrm{E}$, VII 814 B, XII 952 E), dann werden Wölfe erwähnt, vereinzelt Löwe Gorg. 483 E), Schwein (Staat VII 535 E), Ziegen (Gesetze I 639 A), Hahn (Theät. $164 \mathrm{C}$ ), Tauben (Theät. $197 \mathrm{C}$ ), Ameisen und Frösche (Phädon 109 B), Grillen (Symp. $191 \mathrm{C}$ ), Fische (Phädon $109 \mathrm{E}$ ), Krampfroche (Menon $80 \mathrm{~A}$ ). Auch hier bekundet sich eine fast wissenschaftliche Gründlichkeit des Beobachtens. - An letzter Stelle nun kommen die Gleichnisse aus der Natur. Sechs von diesen, und zwar besonders charakteristische, stehen im Phädon. Die Seele verläßt derı 


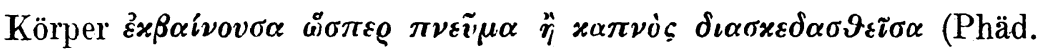
$70 \mathrm{~A}$ ), weiter ausgeführt ist dieser Gedanke Phäd. $77 \mathrm{D}$; es ist das kaum ein Gleichnis zu nennen, denn es ist tatsächlich Vorstellung des primitiven Menschen. Ein sicher beobachtetes, lokal bestimmtes

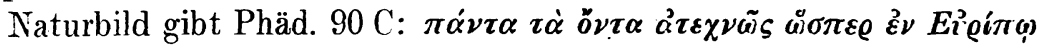

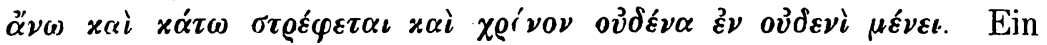
Gleichnis vom Meer bietet auch Phäd. 109 C: wir glauben oben auf der

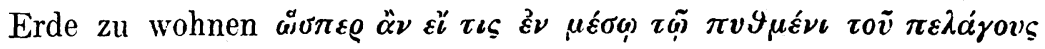

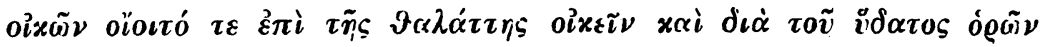

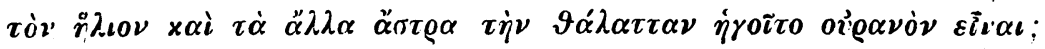
hier ist eine charakteristische Verbindung von naturwissenschaftlicher Erfahrung und mystischer Phantastik, ebenso Phäd. $110 \mathrm{~A}: \ddot{\eta} \delta \varepsilon \mu \dot{\varepsilon} \nu$

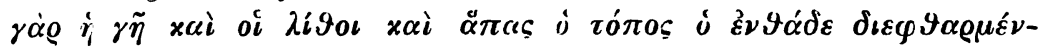

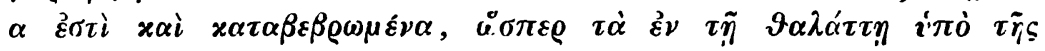

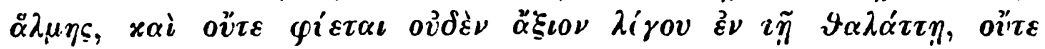

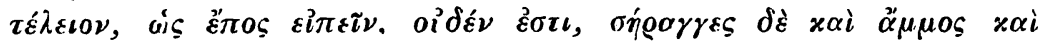

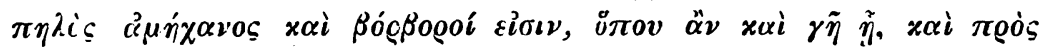

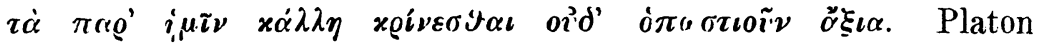
durchforscht die Erscheinungen der Natur, als Künstler aber ist er mit der Erfahrungswelt nicht zufrieden, er konstruiert schönere, harmonischere Bilder in seiner Phantasie. Im Phädon stehen noch zwei Gleichnisse, die eingehende Naturkenntnis beweisen: $111 \mathrm{E}$ werden die unterirdischen Feuerströme beschrieben

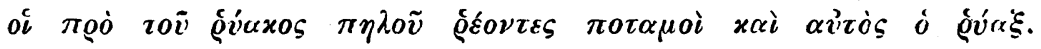
Platon hat mehrmals in Sizilien geweilt und war jedenfalls über den Ätna genau unterrichtet. Grandioser, gefühlsmäßiger haben ca. 100 Jahre vorher Äschylus (Prometh. 367 ff.) und Pindar (Pyth. 1, 21 ff.) das gewaltige Naturereignis eines Vulkanausbruches gemalt. Auf ein eigenartiges Phänomen, eine Sonnenfinsternis, deutet Phädon $99 \mathrm{D}$ : wer sich auf die Sinneserkenntnis verläßt, der erleidet dasselbe

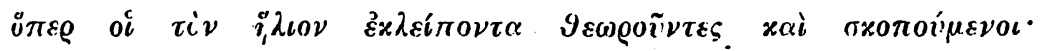

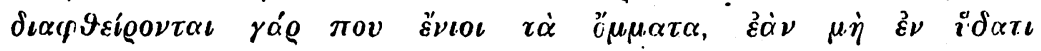

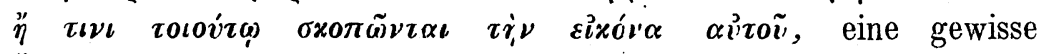
Ähnlichkeit hiermit zeigt Ges. X 897 D. Derartige Gleichnisse wie im Phädon treten in anderen Dialogen nur. vereinzelt auf, bloß im Staat finden sich gleichfalls sechs, aber der Staat ist auch um so viel umfangreicher. Phädrus $255 \mathrm{C}$ wird von der Wahrnehmung

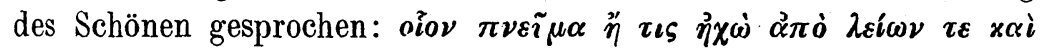




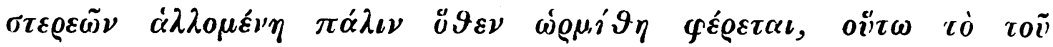

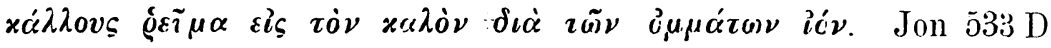

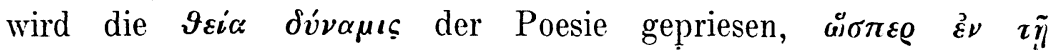

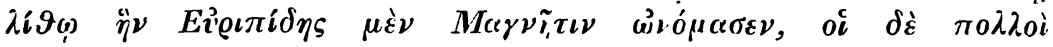

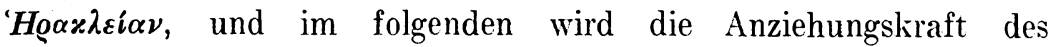
Magneten verdeutlicht. Ein ziemlich ausgeführtes Gleichnis ist

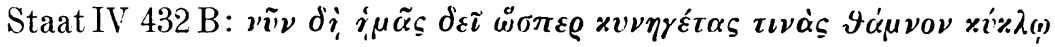

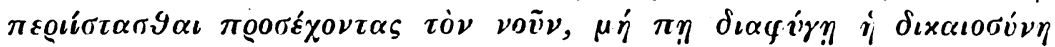

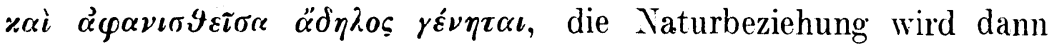

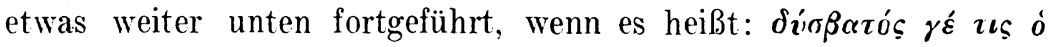

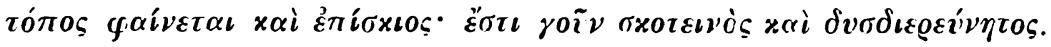
Hier ist die Szenerie zwar deutlich gekennzeichnet, aber die Schilderung ist doch nicht stark gefühlsmäßig betont. Sehr häufig sind namentlich die Beziehungen zu Wasser und Schiffahrt in Gleichnissen, Vergleichen

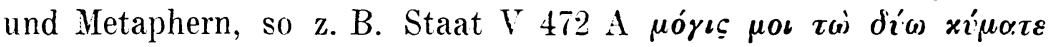

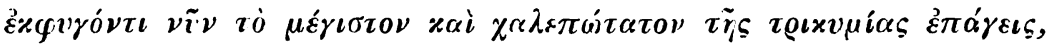

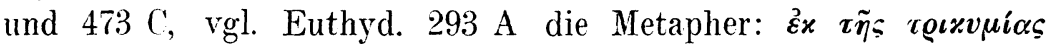

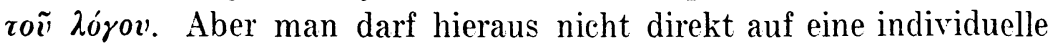
Vorliebe schließen: derartige Bilder lagen dem Griechen und speziell dem Athener außerordentlich nahe, und sie treten daher überall in der Literatur auf. Origineller ist darum ein Gleichnis wie Staat VI

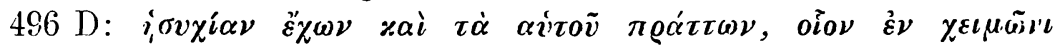

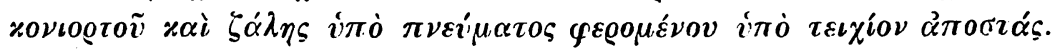
Das grandioseste und am weitesten ausgeführte Gleichnis im Staat ist aber das bekannte Höhlengleichnis am Anfang des VII. Buches. ঠ̃14 A ff. Hier ist das Naturgefühl in eigenartiger Weise in Phantasiebilder aufgegangen; es ist interessant zu sehen, wie der Künstler die Faktoren des Naturgeschehens frei konstruktiv verwertet, um ein Gebilde zu schaffen, das zwar in der tatsächlichen Wirklichkeit nicht anzutreffen ist, das aber doch alle Wahrheit des ästhetischen Scheins besitzt. Bemerkenswert ist auch die Hervorhebung der Lichtwirkung, wie sie in der Antike nicht häufig anzutreffen ist und bei Platon nur in der Zeit seiner ausgereiften Kunst. Man erhält hier wirklich den Eindruck eines Naturgemäldes. Von den übrigen Dialogen bieten noch Timäus und Gesetze einzelne Naturgleichnisse (3 und 4). Zwei Gleichnisse des Timäus beziehen sich auf die Pflanzenwelt: 86 C;

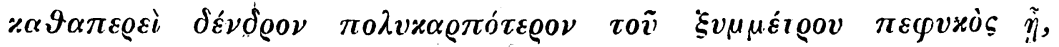

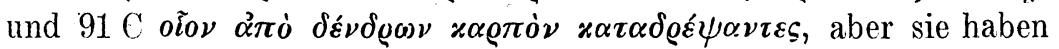


kaum persönliche Färbung. In den Gesetzen IV $719 \mathrm{C}$ wird vom

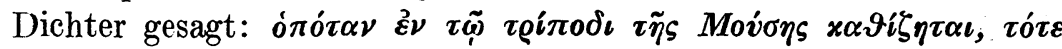

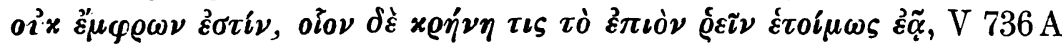
heißt es im Hinblick auf die Bevölkerung der zu gründenden Stadt:

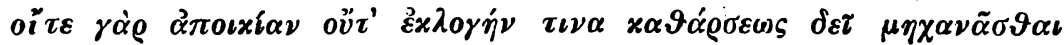

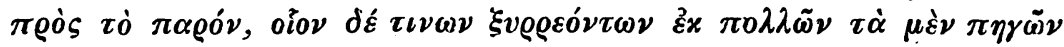

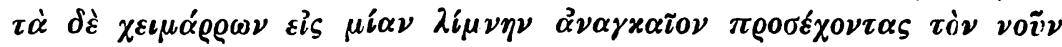

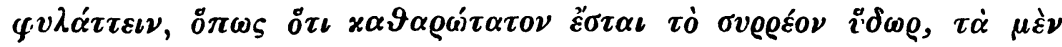

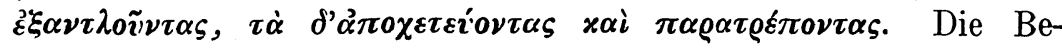
ziehung auf das Praktisch-menschliche tritt bei den meisten Gleichnissen hervor; reine Naturgleichnisse sind also ziemlich selten, und ein besonderes ästhetisch-gefühlsmäßiges Verhältnis wird kaum a ngedeutet.

$\mathrm{Zu}$ ähnlichen Ergebnissen gelangen wir bei der Betrachtung der kürzeren V e r g l e i c h e, die meist nur aus einem oder zwei Worten bestehen. Auch hier sind weitaus am häufigsten die Bilder aus dem praktischen Leben der Menschen genommen. Von 150 entstammen nur 18 dem Tierleben und 21 der Natur. Die mancherlei Beschäftigungen und Tätigkeiten der Menschen werden zum Vergleich herangezogen, hauptsächlich aber einzelne Gegenstände, die irgendwie dem praktischen Gabrauche dienen. Mehrmals findet sich z. B. der Vergleich mit dem Spiegel, wie Phädr. 255 D, Theät. 193 C, 206 D, Tim. 72 C, Ges. X 905 B. Auf die Vergleiche aus der Schützenkunst habe ich schon bei Besprechung der Gleichnisse hingewiesen: Symp. $219 \mathrm{~B}$, Phileb. $23 \mathrm{~B}$, Gas. IV $705 \mathrm{E}, 717 \mathrm{~A}$. Andere Vergleiche beziehen sich auf die Schiffahrt: Staat III 389 C, Tim. 85 E, Kritias 109 C, Ges. XII $961 \mathrm{C}$; originell sind besonders solche mit einer gewissen komischen Tendenz wie in der Rede des Aristophanes Symp. 190 D, 193 A, ebenso ist das Komische beabsichtigt Phäd. $99 \mathrm{~B}$, auch wohl Tim. $70 \mathrm{E}$, $78 \mathrm{~B}$. Von den Vergleichen aus dem Tierleben sind einzelne ganz un-

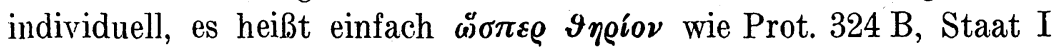

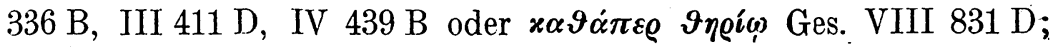
sonst werden erwähnt Pferde (Ges. IV 708 D), Hunde (Staat V 466 D, VII 537 A, 539 B, Ges. II 654 E), Bienen (Staat VII 520 B, VIII 552 C), Schlange (Staat II 358 B), Natter (Symp. $217 \mathrm{E}$ ), Schaltier (Phädr.

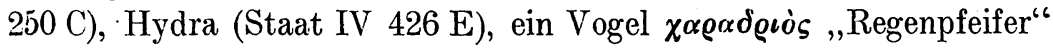
(Gorg. 494 B). - Auch die Vergleiche aus dem Naturleben sind kurz und verraten keine Beziehung auf persönliches Gefühl. Relativ die

Archiv für Geschichte der Philosophie. XXIV. 2. 12 
meisten finden sich wieder im Phädon (6), dann in Timäus (5), Staat (5), Gesetzen (7). Bilder, die sich auf das Wasser beziehen, sind auch

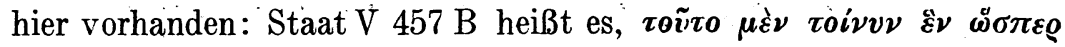

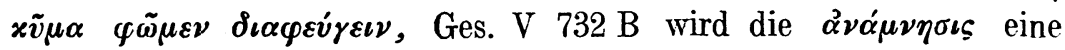

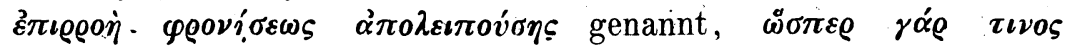

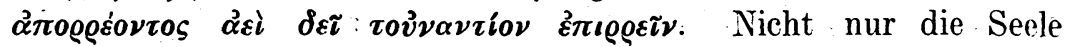
ist $\omega \sigma \sigma \pi \varepsilon \varrho \pi \nu \varepsilon \tilde{\mu} \alpha$ (Phäd. $70 \mathrm{~A}$ ), sondern auch von der Rede gilt derselbe Vergleich Staat III 394 D. Die Seele wird auch mit dem Samen

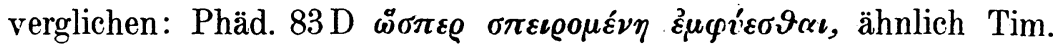
$73 \mathrm{C}$, Ges. IX $853 \mathrm{D}$. Von den Adern heißt es Tim. $77 \mathrm{C}: \tau \dot{\delta} \sigma \tilde{\omega} \mu \alpha$

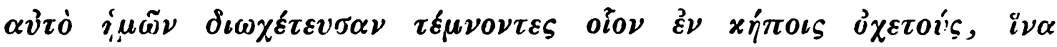

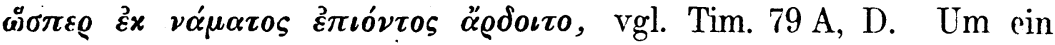
apathisches, gefühlloses Leben zu bezeichnen, wird Gorg. $494 \mathrm{~A}$ der

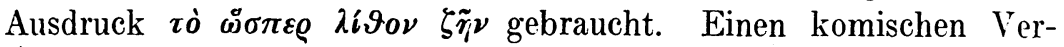

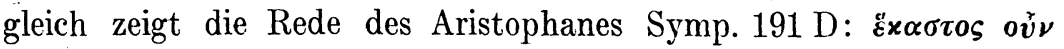

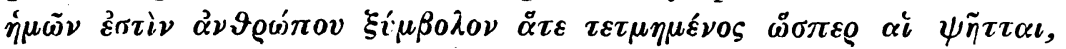

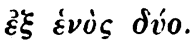

Von einzelnen metaph oris che $\mathrm{n}$ orten will ich nur die charakteristischsten aufzählen. Am zahlreichsten wohl sind die Metaphern von der Jagd: Phäd. 66 A, C, Phädr. 262 C, Phileb. 64 E, Gorg. 489 B, Euthyd. 295 D, Soph. 235 B. Phädr. 268 A werden die Reden ein Gewebe भi่ œíov genannt; Parm. $137 \mathrm{~A}$ heißt es $\delta \iota \alpha \nu \varepsilon \tilde{v} \sigma \alpha \iota$

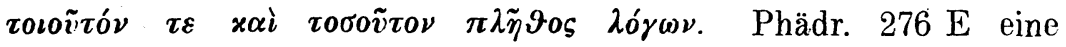

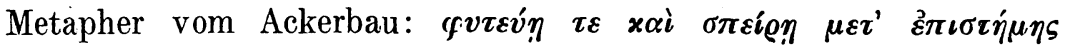

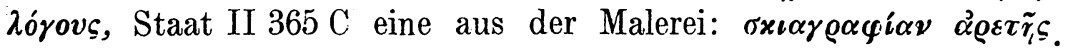
Aus dem Leben der Bienen stammen die metaphorischen Worte:

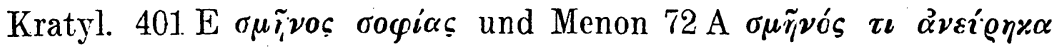

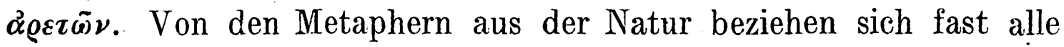

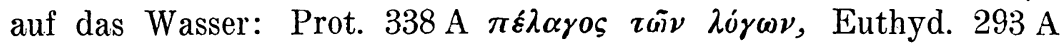

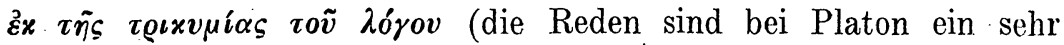
beliebter Gegenstand des Vergleichs), Ges. V $740 \mathrm{E} x \tilde{v} \mu \alpha \varkappa \alpha \tau \alpha x \lambda v \sigma \mu \dot{o} \nu$

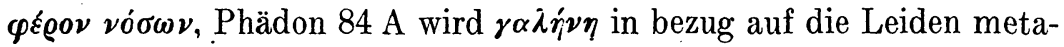

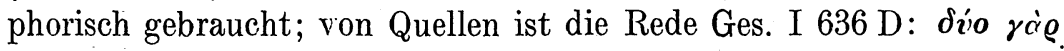

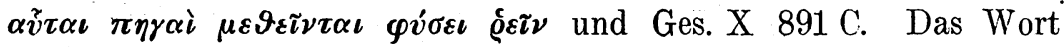

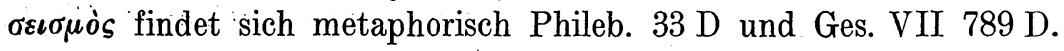

Wenn schon hier die Beziehung zur Natur ziemlich schwach ist, so tritt sie noch mehr zurück in den Beispiele $n$, bei denen eben das Gefühlsmäßige fast ganz ausgeschlossen ist. Sie bieten meist 
die Exemplifizierung eines abstrakten Gedankens durch einen konkreten Fall, sie weisen etwa, um eine philosophische Wahrheit zu demonstrieren, auf ein Gebiet menschlicher Tätigkeit hin, oft nur so, daß einfach der Name des betreffenden Handwerks, der Kunst usw. genannt wird. Von ca. 600 Beispielen stammen nur 32 aus dem Leben der Tiere, 21 aus der Natur. Weitaus am häufigsten wird auf eine bestimmte berufliche Tätigkeit, ein Handwerk usw. Bezug genommen. Am zahlreichsten sind die Hinweise auf die Ärzte und ihre Kunst (66 mal). Dann folgt nach der Häufigkeit der Beispiele die Musik in ihren verschiedenen Ausgestaltungen und Beziehungen zur menschlichen Tätigkeit (Flötenspieler, Leierspieler, Leiermacher, Zitherspieler usw.) (ca. $50 \mathrm{mal}$ ), schon in weiterem Abstand kommen die bildenden Künste (Malerei, Bildhauerei usw.) (ca. 30 Beispiele), die Dichtkunst wird nur et wa 12 mal erwähnt. Dem Gebiet der Mathematik sind etwa 35 Beispiele entnommen, davon ca. 15 der Geometrie. Im übrigen werden besonders handwerksmäßige Beschäftigungen häufig angeführt: so Schuhmacher (25 mal), Steuermanī (22 mal), Zimmermann (21 mal), Landmann ((21 mal) und so die verschiedensten Berufe und Stände: Baumeister, Stratege, Weber, Koch, Krämer, Händler, Bankier, Schiffbauer, Schmied, Töpfer, Hirt, Krieger, Lehrer, Richter, Fischer, Wagenlenker, Mauleseltreiber, Räuber; vereinzelt: Gerber, Walker, Färber, Bader, Bäcker, Peltast, Herold, Ofenmacher, Ziegelstreicher, Puppenmacher, Hebamme, Wahrsager, Taschenspieler, Zauberer. Auch auf Sport und Spiel beziehen sich mehrere Beispiele, namentlich auf die Reitkunst (17) und Gymnastik (16), dann Jägerei und Schützenkunst, Tauchen und Schwimmen, Wettlauf; mehrmals wird auch das Brettspiel genannt. - Von den Tieren werden hauptsächlich wieder Pferde und Hunde erwähnt, dann auch vereinzelt Esel, Rinder, Schafe, Löwen, Hirsch, Panther, Eber, Schwein, Hahn, Wachtel, Polyp, Kaulquappe. Dreimal treten Vögel in den Gesetzen auf: da auch bei den Gleichnissen die Vögel in den Gesetzen bevorzugt werden, dürfen wir wohl hier auf eine Liebhaberei des alternden Platon schließen; auch als Geschenke für die Götter werden Vögel empfohlen Ges. XII 956 B. - Bei den Beispielen aus der Natur werden meist nur die einzelnen Objekte aufgezählt: Schnee und Feuer, Wind, Meeresstille, Wasser, Meer, Bäume, Himmel, Sonne, Sterne, Samen und Wurzeln, Reif und Frost, Stein, Holz, Eisen. Staat III $396 \mathrm{~B}$ wird gefragt, in bezug auf die Dichter: $i \pi \pi$ ovs 


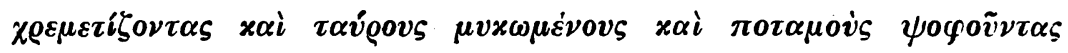

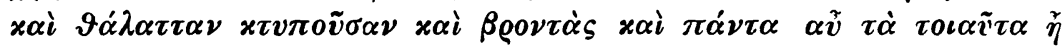
$\mu \iota \mu \eta_{\sigma o \nu \tau \alpha \iota}$, vgl. auch $397 \mathrm{~A}$. Kritias $107 \mathrm{C}$ werden als Objekte der

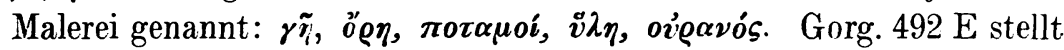
Kallikles die Steine und die Toten nebeneinander, weil sie am glücklichsten seien, wenn Glück gleich Bedürfnislosigkeit wäre. Gorg. 468 A werden als Beispiele von Objekten, die weder am Guten noch am Bösen teilhaben, Steine und Hölzer bezeichnet.

Wenn wir nun die verschiedenen Mittel der Veranschaulichung zusammennehmen, wie wir sie bisher betrachtet haben, so erhalten wir etwa die Zahl von 800 Bildern; davon entstammen 80 dem Tierleben, 74 der Natur. Zweifellos steht hier das menschliche Leben mit seinen verschiedenen Formen und Beziehungen im Vordergrunde des Interesses. Von den Zuständen und Tätigkeiten des Menschen, . vor allem von den praktischen Beschäftigungen her werden die Bilder genommen. Der primitive Mensch denkt und handelt nach utilitarischen Gesichtspunkten, und auch bei den Griechen ist das Praktische noch fast ausschließlich maßgebend. Die Vorgänge des täglichen Lebens, die Beschäftigungen, die Gebrauchsgegenstände, das sind die ersten Objekte, auf die das Auge des Menschen trifft und die sein Denken und Fühlen beeinflussen. Der Grieche in der klassischen Zeit steht zwar nicht mehr auf jener ersten Stufe, wo das physische Bedürfnis das Hauptmotiv ist, sondern sein Geist hat sich die Freiheit errungen, selbständige Gabilde zu schaffen und sich über den engen Horizont des Praktischen zu erheben, aber seine Anschauung ist doch noch gefangen in dem unmittelbaren Erleben des täglichen Treibens, und es wird ihm schwer, die praktische Tendenz ganz abzustreifen. Platon nun erfaßt mit scharfem Blick die konkrete Gegenständlichkeit der äußeren Wirklichkeit, er beobachtet bis ins kleinste das Leben und Tun der Menschen, aber das gewaltige theoretische, geistige Prinzip in ihm unterwirft sich dann diese Interessen und macht sie seinen Gedanken und Problemen dienstbar. In der Neuzeit werden die poetischen Bilder nicht mehr in diesem Umfang wie bei der Antike aus dem praktischen Leben, namentlich nicht so sehr aus dem Gebiet von Handwerk und Gewerbe entnommen, weil das Geistige sich zu größerer Selbständigkeit entwickelt hat, weil Wissenschaft und Kunst sich schärfer abgegrenzt haben gegenüber anderen Bereichen, weil geistig. tätige Menschen meist nicht mehr jene enge Berührung mit dem All- 
täglichen und Praktischen besitzen. Auch die Beziehungen zur Tierwelt sind im Altertum noch stärker als heute. Das Tier tritt ja auch schon früh in den Interessenkreis des Menschen ein, er kämpft mit ihm, er zähmt es, und so treibt ihn das praktische Bedürfnis, das Tierleben zu beobachten und verstehen zu lernen. Wenn bei Platon noch die Bilder aus dem Tierleben überwiegen über die aus der Natur, so ist bei den meisten neueren Schriftstellern und Dichtern sicher das Umgekehrte der Fall. Am charakteristischsten vielleicht für das innige Verhältnis zur Tierwelt ist die humorvolle Stelle im Staat $563 \mathrm{C}$, wo auch die Hunde, Pferde und Esel von der Idee der demokratischen Freiheit angesteckt gedacht werden, so daß sie frei und vornehm einherlaufen und keinem ausweichen. Die Natur im engeren Sinne ist für den Menschen lange Zeit etwas Fremdes, Unfaßbares, Dämonisches, und erst allmählich gelangt er dazu, auch die elementaren Gewalten zu bändigen und praktisch zu verwerten. Auch hier bringt der Nützlichkeitsstanıpunkt zuerst den Einzelnen in eine nähere Verbindung mit der Außenwelt. Das rein ästhetische Verhalten gegenüber der Natur tritt erst auf einer viel höheren Entwickelungsstufe ein, wo der Mensch sich seiner Subjektivität und Geistigkeit so weit bewußt ist, daß er die Objekte subjektiviert und doch ihre Gegensätzlichkeit und Eigenwertigkeit anerkennt. Der Grieche konnte noch nicht jenes subjektive Gefühlsverhältnis zur Natur besitzen, wie der moderne Mensch, da er noch nicht die ungeheure Macht der Subjektivität und Individualität kannte, da er sich selbst noch riel mehr als Naturprodukt fühlte und nicht als ein eigenartiges geistiges Wesen, .das der Natur gegenübersteht, das ihr entfremdet ist und sich nach ihr zurücksehnt, sein Naturgefühl ist also weit ehẹr naiv als sentimentalisch zu nennen. Das schließt nicht aus, im Gegenteil, es ist durch seine Richtung auf das Konkrete bedingt, daß er die einzelnen Naturobjekte und Vorgänge genau beobachtet und zu seinem eigenen Erleben in Beziehung setzt, aber das komplizierte, mannigfach abgetönte Gebilde einer Landschaft etwa kann er nicht wiedergeben, die eigenartig persönliche, malerische Färbung, der Hauch subjektiver Seelenhaftigkeit fehlt seinen Schöpfungen noch fast ganz, weil er das noch nicht bewußt empfindet und fühlt. Bei Platon sprechen in seinem Verhältnis zur Natur zweifellos künstlerisch-ästhetische Momente mit, aber auch bei ihm sind sie noch nicht zur Herrschaft gelangt. In seinen Bildern und Gleichnissen 
zeigt sich doch noch häufig die praktische Bedeutung der Naturobjekte, und vielfach ist die Natur nur ein Mittel oder die Staffage für den Menschen und seine Tätigkeit. Allerdings wird in manchen auch das Naturgeschehen für sich betrachtet, und da ist wohl ästhetische Absicht anzunehmen. Platon erscheint in den Bildern als ein scharfer Beobachter des Einzelnen, ja er sucht manchmal direkt besondere wissenschaftlich interessante Phänomene aufzuweisen, dagegen verschmäht er eine allgemeine, gefühlsmäßige Charakteristik. Kaum wird bei einem Bilde oder G'eichnis die Schönheit der Formen an Naturobjekten oder die Gioßartigkeit eines Geschehens hervorgehoben. Es liegt das allerdings in den Tendenzen Platons begründet; eine stärkere Betonung des Gefühlsmäßigen würcle ohne Zweifel dem intellektuellen Gehalt schaden. Die Bilder dienen meist der Veranschaulichung der G $\mathrm{G}$ danken, sie sollen ein Abstraktum durch etwas Konkretes erläutern, sie haben also einen bestimmten Zweck, und man darf sie daher nicht als unmittelbaren, lyrischen Gafühlsausdruck ansehen. Die Eigenart Platons zeigt sich oft nicht so sehr in der Art des Gleichnisses als in der Wahl des Gegenstandes, der verglichen wird. .Es ist bewundernswürdig, wie abstrakte Vorstellungen durch einen, wenn auch noch so individuellen Vergleich beleuchtet werden. So erhalten auch abgeblaßte Bilder eine gewisse neue Färbung durch Beziehung auf verschiedenartige Objekte. Gerade dadurch, daß die Gleichnisse oft ganz gebräuchlich, typisch sind und das Absonderliche, Eigenartige gemieden ist, ergibt sich eine Leichtverständlichkeit und Flüssigkeit der Darstellung. Viele Gleichnisse und Metaphern bei Platon sind für den Griechen volkstûmlich, sie können daher nicht als spezielles Charakteristikum Platons gelten. So namentlich die Bilder von Meer und Schiffahrt usw., die bei griechischen Dichtern, besonders Euripides, noch viel mehr und eigenartiger ausgeprägt sind. Wenn ich so betone, daß die poetischen Bilder für Platon ein stilistisches Mittel sind, so ist damit gesagt, daß sie für das Naturgefühl nur eine sekundäre Bedeutung haben können, daß sich in ihnen nicht die reine, individuelle Beziehung des Ich zur Natur darstellen kann, sondern nur ein allgemeines, äußeres Verhältnis. Ein gewisser Gefühlsuntergrund ist allerdings bei poetischen Bildern in der Regel vorhanden. Namentlich bei den ausgeführten Gleichnissen sind Erinnerungsbilder an Erlebnisse oder Erfahrungen anzunehmen, die in der Seele eine be- 
sondere Stimmungsfärbung empfangen, und èben auf Grund dieser werden sie mit anderen Objekten, die in die Sphäre des Seelischen eintreten, verbunden. Vielfach sind jedoch die Gleichnisse durch den literarischen Gebrauch so fest geworden, daß sie zur Verwendung bereit liegen, ohne durch ein tatsächliches Erlebnis geschaffen zu sein. Für Platon ist charakteristisch, daß er nicht nur Abbilder eines wirklichen Geschehens gibt, sondern daß die Erinnerungsbilder mitunter gleichsam in ihm fortleben und sich weiterentwickeln, sich mit anderen verbinden, sich mit Phantasievorstellungen umkleiden, um als freie künstlerische Schöpfungen zu erscheinen. So entstehen sekundäre Naturbilder, Naturphantasiebilder wie besonders das Höhlengleichnis (Staat VII Anfang). Die Metapher nun bietet eine eigentümliche Verschmelzung von Vergleich und Verglichenem, die Beziehung zwischen beiden ist so eng geworden, daß sie im Gefühl zu einer Einheit zusammengefaßt werden. Es işt das zweifellos eine primitive Form, denn die Sprache ist in ihren hauptsächlichen Elementen metaphorisch und symbolisch. Bei dem primitiven Menschen beruht die Häufigkeit ihrer Anwendung wohl zum großen Teil auf einem Mangel an Ausdrucksfähigkeit, dann aber gerade auf der Freude an der Variation und Neugestaltung von Beziehungen, und so wird sie ein Hauptmittel des poetischen Stils. Aber jene überragende Bedeutung kann ioh dem Metaphorischen nicht zuerkennen, wie es A. Biese tut (,Die Philosophie des Metaphorischen“, Hamburg u. Leipzig 1893). Es ist wohl eine notwendige Form der Anschauung des primitiven Menschen, aber nicht die alleinige und nicht die wesentliche. Biese hat das Verdienst, die Ursprünglichkeit und Selbständigkeit der Metapher gegenüber dem Gleichnis betont zu haben, aber man muß doch darauf hinweisen, daß in der entwickelten kunstmäßigen Dichtung die Metapher vielfach nicht die primäre Form darstellt, sondern durch ein vorhergegangenes Gleichnis bedingt ist. Bei Platon können wir mehrmals ein Nebeneinander von Gleichnissen, Vergleichen, Metaphern derselben Art beobachten, und es scheint mir da meist das Ausgeführtere die frühere, unmittelbare Anschauung zu repräsentieren.

Interessant ist es auch zu sehen, wie die poetischen Bilder auf die einzelnen Werke verteilt sind. Von den Beispielen sehe ich hier $a b$ : sie finden sich fast überall, gar nicht oder äußerst spärlich nur im Kriton, Menexenos, Timäus, Parmenides, Kritias. Gleichnisse, 
Vergleiche und Metaphern sind in mehreren Dialogen nicht oder wenig vorhanden, so im Lysis, Laches, Hippias, Menexenos, Jon, Euthyphron, Kriton, Parmenides, Charmides, Menon, Sophistes und in der Apologie. Am reichsten ist damit der Phädon ausgestattet, wenn ihn auch in bezug auf die absolute Zahl der Bilder die weit längeren Werke, Staat und Gesetze, übertreffen. Immerhin rückt der Staat, auch wenn wir Verhältniszahlen nehmen, ziemlich nahe an den Phädon heran, während die Gesetze zurückbleiben. Ferner treten die poetischen Bilder ziemlich stark hervor in Timäus, Phädrus, Theätet, Symposion, Philebus, Euthydem. Fast die gleiche Reihe erhalten wir, wenn wir speziell die Häufigkeit der Naturbilder untersuchen. Hier steht der Phädon sogar der absoluten Zahl nach an erster Stelle, es folgen dann Staat, Gesetze, Timäus (letzterer bei relativer Zahl direkt hinter Phädon), in den übrigen Dialogen sind Naturbilder nur ganz vereinzelt. Charakteristisch ist, daß Theätet und Euthydem jetzt ganz aus der Reihe herausfallen. Im Theätet finden sich hauptsächlich Beispiele und Sprichwörter, kurze Vergleiche aus dem menschlichen Leben, die stellenweise sogar gehäuft und mit $\ddot{\eta} \ldots \ddot{v}_{i}$ verbunden sind, eine Eigentümlichkeit, die auch in den Gesetzen auftritt. Mehrere lang ausgesponnene Gleichnisse zeigt der Staat, besonders im 6. und 7. Buch. In den Gesetzen sind die Vergleiche meist ziemlich schwach; dasselbe Bild wird mehrmals angewandt, Einzelnes wirkt fast gesucht und manieriert.

Wenn in den poetischen Bildern also nur Spuren von Naturgefühl zu finden sind, so führt etwas weiter die Betrachtung selbständiger Naturschilderungen, wie sie namentlich zur Ausmalung der $\mathrm{Sz}$ enerie in den Dialogen dienen. In bezug auf die Einkleidung und die Weise der Gesprächsführung muß man unterscheiden zwischen einfach dramatischen und referierenden Dialogen. Die szenischen Daten beschränken sich in der Regel auf kurze Orts- und Zeitangaben. Die rein dramatischen Dialoge zeigen überwiegend keine Szenerie: Phädrus und Gesetze bilden eine starke Ausnahme; im Kriton ist die Tageszeit angegeben $43 \mathrm{~A}$, im Menexenos der Ort, woher der eine Sprecher kommt $234 \mathrm{~A}$, ebenso im Jon 530 A, im Euthyphron 2 A wird dazu noch der Ort der Handlung. genannt. In den referierenden Dialogen, die entweder ein Einleitungsgespräch besitzen oder rein diegematisch sind, werden stets kurze szenische Angaben gemacht. Im Phädon ist das Lokal ja ohne weiteres 
bestimmt als das Gefängnis ( $57 \mathrm{~A}$ ), die Zeit erfahren wir aus $59 \mathrm{D}$ und

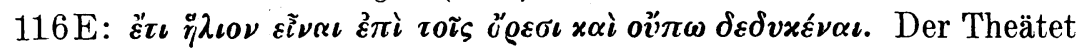
bietet nur eine indirekte Ortsangabe (142 A), und am Schlusse des eigentlichen Gesprächs gibt Sokrates das Ziel seines Ganges an (210 D). Beim Symposion berichtet in der Einleitung der Erzähler:

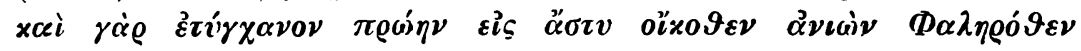
$(172 \mathrm{~A})$, die Zeit des Mahls wird bestimmt durch $173 \mathrm{~A}$; Leben erhält die Szenerie dann besonders durch das wirkungsvolle Bild des hereinbrechenden Alkibiades (212 D), und wenn von ihm gesagt wird, er sei bekränzt gewesen mit einem dichten Kranz von Efeu und Veilchen, so ist das ganz der Stimmung angepaßt. Im Euthydem ist Ort und Zeit kurz bezeichnet $271 \mathrm{~A}$, das Lokal wird dann etwas noch weiter ausgemalt, ja es wird belebt und zu den handelnden Personen in gefühlsmäßige Beziehung gesetzt, wenn es

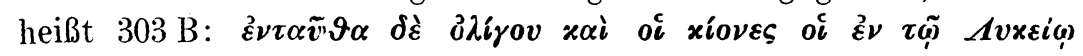

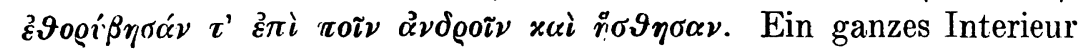
führt uns der Protagoras vor, besonders 314 ff., die Zeit ist genannt 310 A. Von den diegematischen Dialogen hat der Parmenides im Anfang eine Ortsangabe $(126 \mathrm{~A})$, der Charmides Orts- und Zeitangabe $(153 \mathrm{~A})$, etwas reicher ist der Lysis ausgestattet (203 A, B f. und 206 E). Der Anfang des Staates gibt Ort und Zeit (327 A), die Szenerie wird nachher noch' weiter geschildert, besonders $328 \mathrm{~B}$. In der Regel also sind die szenischen Angaben bei Platon kurz und trocken, nur im Phädrus und in den Gesetzen sind sie ausführlicher. Der Phädrus zeigt eine wirkliche Naturschilderung. Im Anfang ist in der üblichen Weise Ausgangspunkt und Ziel angegeben (227 A), die Zeit erfahren wir erst $242 \mathrm{~A}$, allerdings weist schon $227 \mathrm{~A} \vec{\varepsilon} \xi$ $\varepsilon \dot{\omega} \vartheta \iota v o \tilde{v}$ darauf hin. Auf einem Spaziergang also findet das Gespräch statt: schon das ist eigentümlich, denn das zwecklose Lustwandeln im Freien war im Altertum nicht so gewöhnlich wie heute; Phädrus gibt auch den Grund an, weshalb er in diese Gegend komme (227 A), Sokrates spottet leise über längere Spaziergänge (227 D) und wandert selbst wenig (230 D). Näher wird der Naturhintergrund $229 \mathrm{~A} \mathrm{ff}$. geschildert, zuerst allerdings in der praktischen Beziehung auf den Menschen, aber mit deutlicher Freude, und dann äußert sich das

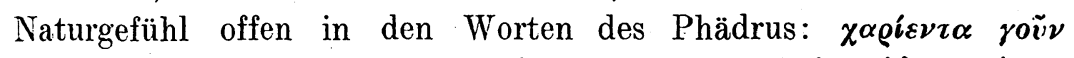

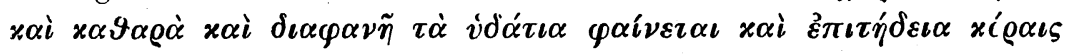
$\pi \alpha i \zeta \varepsilon \iota \nu \pi \alpha \varrho^{\prime} \alpha \imath^{\prime} \tau \dot{\alpha}$. Charakteristisch aber ist, daß sofort Beziehung 
sowenig; denn die Taten des Angeredeten können von der frei schaffenden, ersten Ursache oder der anderen Wesensform herstammen, die diese individuellen Handlungen (in dem Angeredeten) hervorbringt und sie der Anrede entsprechend sein läßt. Es ist also erwiesen, daß Wort und Handlung nicht beweisen, daß ihr Träger lebend und vernünftig ist $\left.{ }^{35}\right)$.

5. In den religiösen Traditionen wird auch berichtet, Gabriel sei dem Propheten in der Gsstalt des Dihja aus dem Stamme Kalb erschienen. Wenn dieses für den vernünftig denkenden Verstand keine Unmöglichkeit enthält, dann ist es ebensowenig unmöglich daß Gabriel in der Person anderer Menschen auftritt. Wenn ich daher mein Kind sehe, so ist es vielleicht nicht mein Kind, sondern Gabriel. $\mathrm{Ja}$, vielleicht ist die Biene, die in der Luft summt, keine Biene, sondern irgend ein Engel. Es ist diese Möglichkeit immer vorhanden, obwohl ich notwendig erkenne, daß sie nicht vorhanden sei.

Durch diese Beweise wurde erwiesen, daß der intuitive Verstand diese Urteile fällt, obwohl sie hinfällig sind. Dann aber sind sie unsicher und nicht allgemein gültig ${ }^{36}$ ). Man könnte entgegnen: Das Urteil des Verstandes über diese Erfahrungstatsachen ist ein abgeleitetes, nicht ein primäres. Jedoch entgegnen wir: Wäre dies der Fall, dann könnten die genannten Urteile nur von dem gefällt werden, der die Ableitung kennt. Ist dies nun aber nicht der Fall - Knaben, Verrückte und Nichtphilosophen fällen ebenfalls diese Urteile dann wissen wir, daß es sich um primäre, nicht spekulativ deduzierte Urteile handelt. Beobachten wir uns selbst, so finden wir in uns ein

$\left.{ }^{35}\right)$ Tusi: „Die orthodoxen Theologen (Mutakallimun) lehren: Eine geordnete Rede, die ein Mensch hervorbringt, beweist mit Sicherheit, daß er lebend und vernünftig ist. Durch das Vorgebrachte wird dies nicht widerlegt. Ferner ist unbestritten, da $B$ geordnete, planmäßige Handlungen einen erkennenden und frei wollenden Urheber haben. Das Vorgebrachte ist also weder nach der Lehre der Theologen noch der der Griechen beweiskräftig."

${ }^{36}$ ) Tusi: „Die Forscher unter den Theologen und Religionsstiftern lehren: Was ein Berichterstatter bezeugt, ist wahr. Wenn es also nicht unmöglich ist, urteilen wir, es sei richtig, und überlassen es der Hand des mächtigen und frei wollenden Schöpfers. Ist es aber unmöglich, so nehmen wir unsere Zuflucht zu einer allegorischen Erklärung, die mit den Prinzipien unserer Religion im Einklang steht, oder wir enthalten uns des Urteils. Sicher ist, daß die definitiv urteilende Erkenntnis durch diese nichtigen Vermutungen nicht ins Wanken kommt." 
bestimmtes Wissen, das besagt: Der zuerst gesehene Zaid ist identisch mit dem später gesehenen, und es ist ebenso richtig und sicher zu behaupten: der erste wurde vernichtet und ein Doppelgänger trat an seine Stelle, als zu behaupten: das Ding ist entweder existierend oder nicht existierend ${ }^{37}$ ).

\section{B e w e i s.}

Die eingehende Beschäftigung mit den Geisteswissenschaften zeigt, daß sich manchmal zwei kontradiktorische Beweise in einem einzigen wissenschaftlichen Probleme einstellen. Der Verstand vermag dann keinen von beiden zu widerlegen und zwar entweder nie oder nur unter gewissen Umständen nicht. Dieses Unvermögen tritt nun aber nur dann ein, wenn der Mensch gezwungen ist, alle Voraussetzungen der beiden Beweise anzuerkennen. Dabei ist es zweifellos, das einer von beiden falsch sein muß; sonst beständen zwei kontradiktorische Gegensätze zurecht. Dies beweist, daß das intuitive, primäre Erkennen manchmal über Dinge urteilt, die nicht in seinem Bereiche liegen.

\section{B e w e is.}

Manchmal urteilt der Mensch mit subjektiver Sicherheit über die Richtigkeit aller Prämissen eines bestimmten Beweises. Darauf wird ihm ein in irgend einer Prämisse übersehener Fehler klar. Aus diesem Grunde geht mancher von einem Systeme zum anderen über. Das erste bestimmte Urteil über die Richtigkeit jener Prämissen war also irrig. Daraus ist ersichtlich, daß der mit Bestimmtheit über evident erscheinende Dinge urteilende Verstand sich täuschen kann ${ }^{38}$ ).

$\left.{ }^{37}\right)$ Tusi: „Diese Ausführung beweist, daß die Widerlegung der evidendsten Erkenntnisse auf Grund der angeführten Schwierigkeiten das sichere Urteil des Verstandes in keiner Weise anficht."

$\left.{ }^{38}\right)$ Tusi: „Wenn der Geist mancher Menschen nicht fähig ist, das Wahre vom Falschen zu unterscheiden und wenn sie gläubig den Lehren folgen, die sie von ihren Vätern und Lehrern empfangen haben, so beweist dies nur, daß sie eine gute Meinung von ihnen haben. Es widerlegt aber nicht die ersten Prinzipien des Erkennens. Ferner läßt der Zweifel an s e k u n d ä r e n Erkentnnissen nicht die beiden Beweise (das Für und Wider) als gleichwertig erscheinen (vgl. auch die griechischen Sophisten bes. Carneades). Ebensowenig ist das Übergehen von einem System zu einem anderen, weil einer von zwei sich anfänglich gleichstehenden Beweisen das Übergewicht erhält, eine 
dafür, daß bei Platon im Alter das unmittelbare Gefühlsverhältnis zwischen Ich und Natur an Intensität verloren hat und mehr das Gedankliche, Symbolische betont wird, wenn auch der Sinn für die objektiv-gegenständliche Schönheit vielleicht verfeinert ist.

Außer in diesen szenischen Einleitungen verweist Platon auch in den eigentlichen Erörterungen der Dialoge mehrfach auf Naturobjekte und Naturgeschehen und gibt mehr oder weniger ausgeführte Schilderungen, in denen sich das Naturgefühl äußern kann. Symp. 196 B preist die Schönheit der Blumen, wenn von Eros gesagt wird:

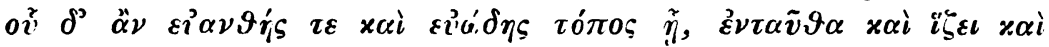
$\boldsymbol{\mu} \varepsilon \dot{v \varepsilon \varepsilon . ~ D i e ~ R e i z e ~ v o n ~ G a ̈ r t e n ~ w e r d e n ~ m e h r f a c h ~ e r w a ̈ h n t, ~ s o ~ h e i ß t ~}$

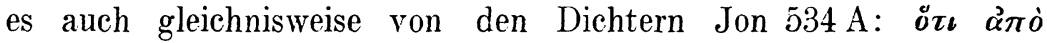

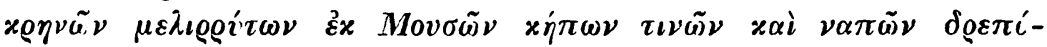

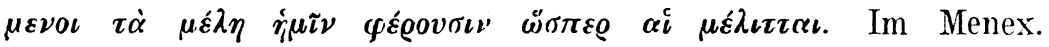
237 C ff. wird die Vortrefflichkeit des Heimatlandes hervorgehoben, und als großer Vorzug gilt es, daß zur Zeit, als es überall auf der Erde nur wilde Tiere und Pflanzen gab, Attika den Menschen als das höchste aller Wesen hervorgebracht habe. Dann wird das Land mit einem Weib verglichen: wie die gebärende Frau die erste Nahrung für das Kind selbst erzeugt, so trägt die Erde Weizen und Gerste als schönstes und bestes Nahrungsmittel für das Menschengeschlecht, außerdem das Öl, welches $\pi \dot{\nu} \nu \omega \nu$ $\varrho \omega \gamma \dot{\eta}$ genannt wird; diese Analogie gilt als Beweis, daß die Erde dem Menschen auch geboren habe,

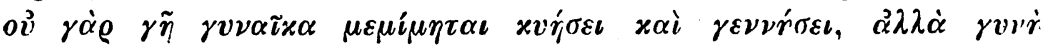
$\gamma \tilde{\eta} \nu$. Die Natur ist hier also anthropomorphisiert, und der Mensch ist ihr höchstes Produkt. Erst durch die Kultivierung und Nutzbarmachung für den Menschen erhält sie einen Wert. - Eine wirkliche geographische Naturbezeichnung ist dann im Timäus enthalten, $21 \mathrm{E}$ ff. ist die Rede von Ägypten und dem Nil, die praktische Bedeutung wird betont, und die tatsächlichen Verhältnisse werden mit Mythen in Verbindung gebracht $22 \mathrm{D} \mathrm{f}$. Ges. III $682 \mathrm{~B}$ wird die Lage von Troja beschrieben, die große schöne Ebene wird erwähnt, die mäßige Anhöhe, auf der es gegründet ist, und die vielen Flüsse, die vom Ida kommen. Bei der geplanten Stadtgründung in den Gesetzen wird dann auch eingehend die geographische Lage berücksichtigt (IV 704 A ff.). Die Stadt soll nicht am Meer liegen, da der Seeverkehr einen schlechten Einfluß auf den Charakter der Bewohner ausübe, nur das Notwendige bringt das rauhe, gebirgige Land hervor, Wald- 
bäume wachsen wenig in der Gegend (705 C), es fehlt daher auch glücklicherweise an Schiffsbauholz. Lage und Klima sind von großer W'ichtigkeit, Veränderungen in der Natur können Umgestaltungen der Lebewesen zur Folge haben (782 A, vgl. Politikus 271 C). Gegen die elementaren Gewalten der Natur müssen Schutzmaßregeln getroffen werden (761 A ff.). Beschädigungen der Natur werden bestraft, wenn sie sich gegen das Eigentum anderer richten (843 D f.). Eingehend wird die Wasserversorgung berücksichtigt, und die Anlagen zu diesem Zweck sollen nicht nur vom Nützlichkeitsstandpunkte aus errichtet sein, sondern sie sollen verschönert werden, um das Schmuckbedürfnis

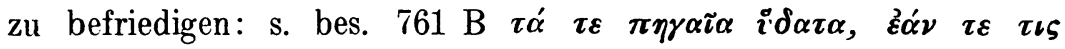

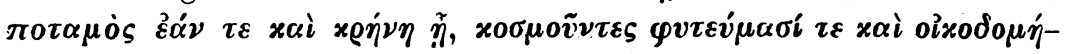

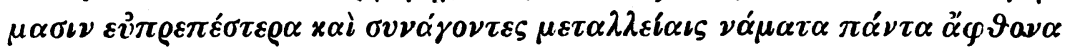

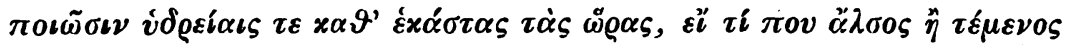

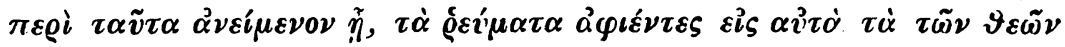

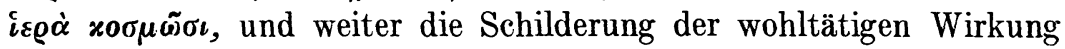
des Badens auf die Greise. W ila mow it z sagt zu dieser Stelle (,Aristoteles und Athen“ I S. 237): „Der Greis, der über die Realität der Zahl grübelt und in bitteren Stunden selbst über den Teufel (die böse Weltseele), dem alles irdische Treiben ein Spiel wird, hat doch seine Jugend nicht verleugnet, da der Knabe auf den Hügeln des oberen Kephisostales an den Quellen von Kephisia träumte. Ein Landkind ist er gewesen und geblieben." Hier sprechen also deutlich ästhetische Motive mit, hier offenbart sich das künstlerische Naturgefühl. Vgl. noch Ges. 761 D, 763 D, Kritias 117 A. Auch für den Schutz der Tiere spricht sich Platon aus: Fischfang und Vogelstellen ist verboten (Ges. 823 D, E). Die Natur wird in den Gesetzen in erster Linie von ethischen und sozialpolitischen Gesichtspunkten aus betrachtet, die ästhetischen Momente sind sekundär. Es zeigt sich also wohl der Tatursinn, aber auch das konstruktive Bedürfnis Platons, das bei seiner Betrachtung der Natur rege wird und am meisten natürlich in mythischen und naturphilosophischen Partien sich äußert. - Auch bei der Schilderung des Urzustandes von Attika im Kritias $110 \mathrm{D}$ ff. spielt die phantasiemäßige Ausgestaltung eine Rolle, wenn auch von realen Zuständen aus rückwärts geschlossen wird. Gebirge und Flüsse werden mit Namen genannt, besonders die Fruchtbarkeit des früheren Landes wird gepriesen; jetzt sei nur noch ein Strich, ein Überbleibsel

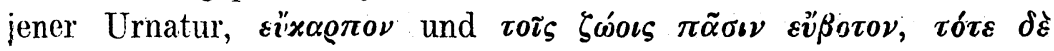




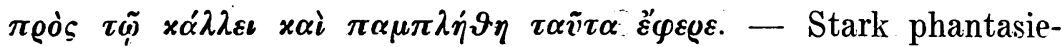
mäßig, mythisch gefärbt ist dann die Schilderung von Atlantis im Timäus und Kritias. Die Lage der Insel wird Tim. 24 Eff. beschrieben. Ein mächtiges Reich blühte dort einst, später aber, als Erdbeben und Uberschwemmungen die Erde verheerten, versank die Insel im Meer, und noch jetzt ist dort die See wegen des tiefen Schlammes unfahrbar; Kritias 108 E weist auf diese Stelle zurück. Dann wird das Land ausführlicher geschildert Kritias $113 \mathrm{C}$ ff. Gerühmt wird die Ebene als

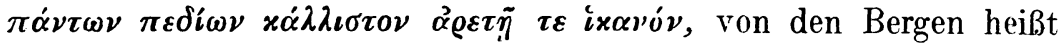
es $118 \mathrm{~B}$, daß sie an Zahl, Größe und Schönheit alle anderen übertreffen. In der ganzen Ausgestaltung zeigt sich aber das mathematische Prinzip, die Formen der Natur sind genau berechnet und verteilt. Die Produkte des Landes werden gepriesen (114 E ff.), die Bodenschätze, die Wälder, bei denen sogleich an ihre praktische Bedeutung für den Zimmermann gedacht wird, die mannigfachen Tiere, die auf Wiesen, in Sümpfen und Flüssen, auf Bergen und in der Ebene ihre Nahrung finden (vgl. auch 118 B). Dann ge-

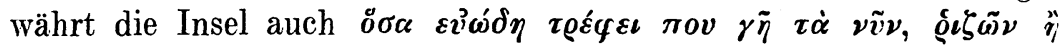

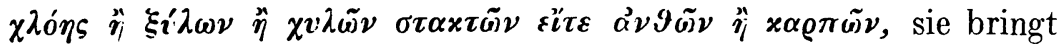
mancherlei Früchte hervor und überhaupt alles, was der Mensch zum Unterhalt braucht. Das Wasser der vielen Quellen wird gelobt (117 A);

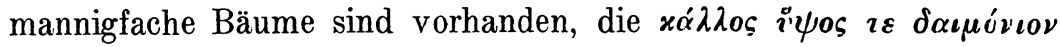

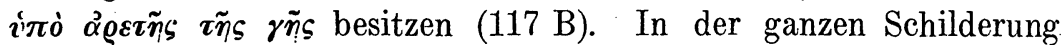
tritt also mehrfach der ästhetische Sinn zutage, die Schönheit der Natur wird empfunden und verwertet zu einem künstlerisch-philosophischen Bild. Die praktische Beziehung wirkt allerdings noch wesentlich mit bei der Betrachtung und Darstellung. Es sind die Elemente aus der realen Natur aufgenommen, aber sie sind gleichsam potenziert in ihrem Schönheitsgehalt, harmonisch gemacht, sie sind mit Phantasievorstellungen umkleidet, um so ein mythisches Ideal repräsentieren zu können. - Das wissenschaftliche Interesse tritt stärker hervor in den naturphilosophischen Ausführurtgen des Timäus. Der Mensch ist hier deutlich als Mikrokosmos gefaßt, der die Elemente des Ganzen in sich enthält, andererseits ist aber auch das Weltall nach Analogie menschlicher Verhältnisse gestaltet. Es steckt viel konstruktive Willkür in dieser Naturphilosophie, und. Th. G o m p e r z sagt mit Recht, Platon leite hier ,,ein ästhetisches oder quasi-ästhetisches Bedürfnis und zugleich die Zuversicht, daß die Natur diesem Be- 
dürfnis überall ein volles Genüge leiste“" Der Kosmos ist $x \alpha \dot{\lambda} \lambda \iota \sigma \tau o \varsigma$

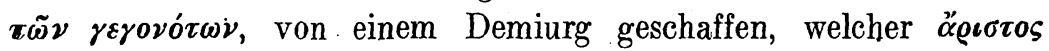

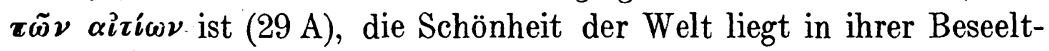
heit begründet $(30 \mathrm{~B})$, nur e in e Welt gestaltete der Demiurg, weil nur eine die möglichst vollkommene sein kann (31 B). Aus den vier Elementen wird der Leib des Weltganzen geschaffen, aber dieser erhält erst durch die unsichtbare Seele Bewegung und Leben; mit dem Himmel und seinen Gestirnen entsteht die Zeit (37 D ff.). Die Gestirne sind göttlich, wie schon mehrere Philosophen vor Platon gelehrt haben und wie Platon selbst auch sonst sagt z. B. Ges. X, $886 \mathrm{D}$.

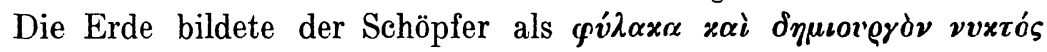

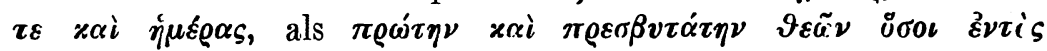

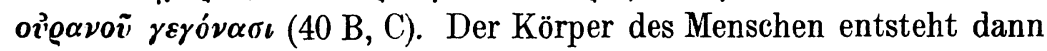

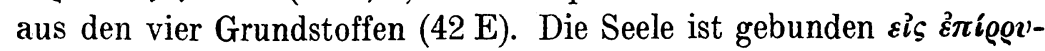

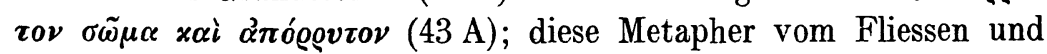
Strömen wird noch weitergeführt, besonders in den Worten $43 \mathrm{~B}$ :

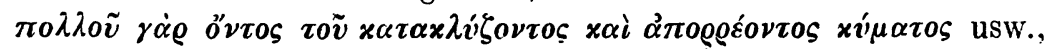
die Leiden der Seele unter den Einwirkungen von außen werden geschildert. Nachdem die Schöpfung und Natur des Menschen dargestellt ist, wird die Untersuchung über das Weltganze wieder aufgenommen. So werden $49 \mathrm{~B}$ die Veränderungen der Grundstoffe besprochen: Wasser verfestige sich zu Stein und Erde, es werde geschmolzen und gelöst zu Hauch und Luft, die entzündete Luft werde zu Feuer; das Feuer kann wieder zu Luft werden, die Luft verdichtet sich zu Wolken und Nebel, sie wird dann zu Wasser, und aus. Wasser entstehen wieder Erde und Steine: ein Kreislauf beherrscht das ganze Naturgeschehen. Auch hier ist ersichtlich wirkliche Beobachtung mit Phantastik gemischt. So werden im einzelnen die Formen und Eigenschaften der verschiedenen Körper behandelt mit philosophischkonstruktiver Auslegung, dann wird hauptsächlich wieder die Natur des Menschen betrachtet. Das Wesen des Menschen ist dem der Naturobjekte bis zu einem gewissen Grad verwandt, aber erst durch die fortschreitende Kultivierung wird ihm die äußere Natur befreundet, erst durch den Ackerbau werden die vorher wilden Bäume und Pflanzen veredelt und in nähere Beziehung zu uns gebracht (77 A). Jene Bevorzugung der kultivierten Natur in ästhetischer Hinsicht, die sich noch deutlicher in häufigen Lobpreisungen von gepflegten Gärten, Parken usw. zeigt, ist typisch für den antiken Menschen. Wenn der Mensch 
die Natur sich dienstbar macht, erschein t sie ihm eben in dem Maße wertvoll, als sie sich seinen Zwecken unterordnet, und das von ihm bearbeitete und verschönerte Stück Natur ist ihm erst durch die aufgewandte Kraft, die er hineingegeben hat, recht schön. Dies Gefühl verallgemeinert sich natürlich und wirkt weiter. Bei Platon nun kommt zu dieser typischen Anschauungsweise noch seine persönliche Vorliebe für gedankliche Konstruktionen, er zwingt der Natur philosophische Ideen auf, er gestaltet sie um nach ethischen, naturphilosophischen und anderen Prinzipien, er bearbeitet sie mit Mythen und Phantasievorstellungen. Dem Griechen gilt die vom Menschen gestaltete Yatur höher als die ungestaltete, und Platon bildet die Natur nicht physisch um, aber er will ihr höhere Schönheit verleihen auf Grund geistiger Ideen. Erst in der Neuzeit, wo der Urzustand so weit zurückliegt, daß wir ihn nicht mehr als schreckhaft empfinden, wo die Yatur durch die fortwährenden Eingriffe des Menschen oft verunstaltet wird, macht sich stärker die Sehnsucht nach der reinen, unkultivierten Natur im Naturgefühl bemerkbar. Wenn Platon hier noch als Vertreter und Vollender überkommener Anschauungen erscheint, so finden wir im Schluß des Timäus doch auch eine andere Seite seines Naturgefühles ausgedrückt. Es wird da noch kurz von der Entstehung der Tiergattungen gesprochen, dann aber wendet sich der Blick noch einmal auf das Weltall, und prächtig endet das Ganze in dem Satz:

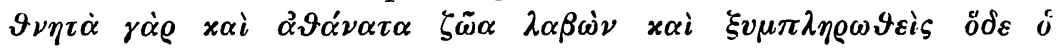

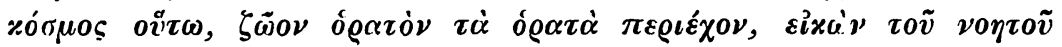

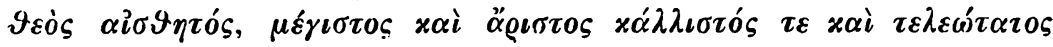

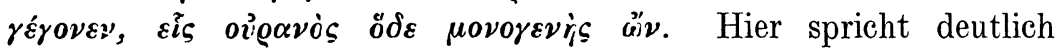
ein Gefühl für die Erhabenheit der Natur, für die Unendlichkeit der Welt. Man fühlt sich an die Worte Kants erinnert: „Zwei Dinge erfüllen das Gemüt mit immer neuer und zunehmender Bewunderung und Ehrfurcht, je öfter und anhaltender sich das Nachdenken damit beschäftigt: Der bestirnte Himmel über mir, und das moralische Gesetze in mir." In einem der platonischen Epigramme, das neuerdings wieder mehrfach für echt gehalten wird, ist das Gefühl für die Größe und Schönheit des Himmels noch eigenartiger, subjektiv-lyrisch gewendet ausgedrückt:

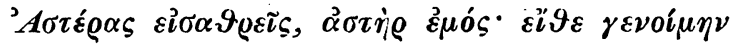

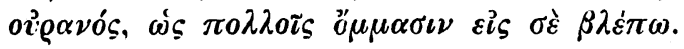


Eine ähnliche Äußerung des aufs Grandiose gerichteten Naturgefühls wird man in der antiken Literatur kaum finden. Platon hat wie Goethe durch seine wissenschaftlichen Studien das Gefühl nicht getötet, sondern mit neuem, feinem Geist belebt. Daß die Gestirne mehrfach als gôttlich gepriesen werden, habe ich schon erwähnt: wenn auch dabei mythologische Vorstellungen maßgebend sind, so spricht doch auch das Naturgefühl mit. Die Sonne wird verherrlicht als das schönste Objekt, als Abbild der Idee des Guten (Staat VI 508 A ff.). Es ist bezeichnend für Platon, daß die Beschäftigung mit Naturwissenschaft und speziell Astronomie sein religiöses Empfinden nur noch steigert, daß er sich in den Gesetzen XII 967 Cff. ausdrücklich gegen die Ansicht verwahrt, die Naturwissenschaft führe zum Atheismus, ja er stellt die Einsicht in das Wesen und Werden der beseelten Welt als eine Grundbedingung des religiös-sittlichen Denkens und Handelns auf.

Wenn wir bisher schon überall reflektive und phantasiemäßige Nomente in die Naturbetrachtung hineingetragen fanden, so ist das noch mehr. der Fall bei den großen es chatologis chen M y t h e n, die ja ein eigentümliches Gemisch von philosophischen, mythischen, religiösen, poetischen und rhetorischen Elementen darstellen. Dabei sind oft auch Naturschilderungen eingewoben, aber es sind Schilderungen einer imaginären, mythisch-phantastisch ausgestatteten Natur. Wie die philosophische Spekulation Platons von der Erscheinungswelt hinführt zu der höheren Welt der Jdeen, so konstruiert er sich über der unvollkommenen äußeren Natur eine schönere, ideale Natur. Wohl noch am meisten an Volksvorstellungen anklingend ist der Jenseitsmythus des Gorgias (523 ff.), wo sich keine

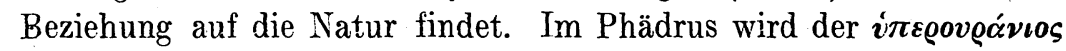
זiло૬ (247 C) zwar nicht näher ausgemalt, aber mit mancherlei poetischen Bildern werden die Schicksale der Seele dargestellt; eine eigentliche Naturschilderung gibt der Mythus nicht, sie wäre bei seinem besonderen $\mathrm{Zweck}$ auch nicht angebracht. Einen großen eigenartigen Mythus enthält der Phädon (108 C ff.). Viele wunderbare Gegenden hat die Erde, aber unsere Sinne vermögen nicht die ganze Schönheit wahrzunehmen. Wir wohnen in den Höhlungen der Erde und sehen alles nur getrübt (109 C f., ein prächtiges Gleichnis). Die Erde selbst aber liegt rein in dem reinen, bestirnten Himmel; was wir gewöhnlich Himmel nennen, ist nur Luft. Wenn aber jemand an die Grenze der Luft käme oder hinauffliegen könnte, dann würde er, wenn seine 
Natur stark genug wäre, die Anschauung zu ertragen, erkennen, daß da der wahre Himmel, das wahre Licht und die wahre Erde sei. Hier bei uns erscheinen alle Dinge verdorben, zerfressen, hier ist nichts Vollkommenes, dort erst werden die Schönheiten offenbar. Dort besteht die ganze Erde aus reinen und glänzenden Farben, wie wir sie hier noch nicht gesehen haben $(110 \mathrm{C})$. Die Schilderung ist so farbenprächtig, daß man gern an die malerische Begabung Platons glaubt. Bäume, Blumen und Früchte wachsen auf dieser Erde, die Gebirge und Steine haben wunderbare Farben, wie sie hier höchstens die Edelsteine zeigen. Auch die Tiere und Menschen sind weit vollkommener. Dort wohnen die Götter wahrhaft in den Heiligtümern. dort sieht man Sonne, Mond und Sterne, wie sie wirklich sind, dort herrscht Glückseligkeit. Die Natur ist idealisiert und ästhetisiert. Ein Kontrastbild zu dieser Schilderung bietet die Beschreibung der Unterwelt. Auch hier wird die ganze Gegend mit großer Anschaulichkeit gekennzeichnet, meisterhaft ist die furchtbare Wildheit gemalt. Man kann nach der Beschreibung ein vollständiges geographisches Bild der Unterwelt entwerfen. Der ganze Mythus im Phädon ist eine glänzende Phantasiekonstruktion eines Naturforschers, Philosophen und Künstlers. - Lange nicht so weit und reizvoll ausgeführt ist der

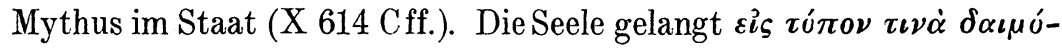

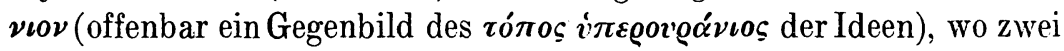
große Schlünde sind; später wird der Ort als Wiese bestimmt, ebenso wie in dem Mythus des Gorgias (Gorg. 524 A), aber näher wird die Szenerie nicht geschildert. Ausführlich werden die Büßungen und Strafen der Seelen beschrieben, dann wird erzählt, wie sie weiter

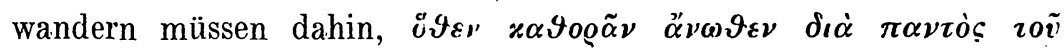

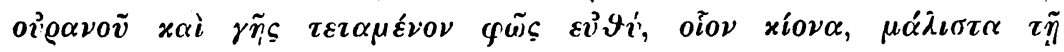

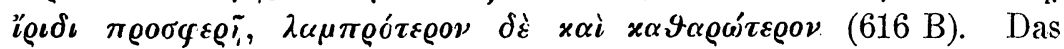
Licht ist das Band des Himmels, welches den ganzen Umfang zusammenhält, an den Enden ist die Spindel der Ananke befestigt, die den Umschwung der Sphären bewirkt. Diese Spindel wird im Detail beschrieben, besonders prächtig sind die $\sigma \varphi c ́(\nu \delta v \lambda o \iota$ mit ihrer Farbenwirkung $(616 \mathrm{E})$. Auf den Kreisen sitzen acht Sirenen, deren Zusammentönen eine Harmonie ergibt. Dazu singen dann die Moiren, die in gleichem Abstand auf Thronen sitzen, weißgekleidet, mit Binden auf dem Haupt. Es wird nun ausgeführt, wie die Seelen selbst sich ihr Los erwählen, wie sie in verschiedene Körper und Zustände eingehen, 
wie aus Menschen Tiere oder aus Tieren Menschen werden können durch die Seelenwanderung. Dann aber erzählt jener wieder zum Leben erwachte Tote, der gleichsam die ganze Schilderung aus Autopsie gibt (621 A ff.), er sei den Thron der Ananke hindurchgegangen und mit anderen zur Ebene der Lethe gekommen, wo furchtbare Hitze herrsche, wo alle Bäume und Gewächse der Erde fehlten, am Abend sei er zum Fluß Ameles mit dem seltsamen Wasser gelangt und habe sich niedergelegt, um Mitternacht aber sei unter Donner und Erdbeben der eine hierhin, der andere dorthin auf die Oberwelt

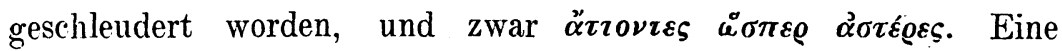
so eingehende poesievolle Naturschilderung wie im Phädon ist hier nicht vorhanden, aber die Eigenart der Szenerie ist doch genügend charakterisiert, in das Phantastische sind immer konstruktiv-wissenschaftliche und ästhetische Momente eingemischt.

Außer jenen großen Mythen finden sich bei Platon noch mehrfache Beziehungen zur Mythologie und Sage, kleinere mythische Szenen und Geschichtchen, bei denen manchmal auch die Natur eine Rolle spielt. So wird im Staat II 359 D ff. die Geschichte vom Ring des Gyges erzählt und dabei die Naturszenerie gemalt, wenn es heißt, es sei ein mächtiger Regenguß und Erdbeben gekommen und ein Spalt entstanden an dem Ort, wo er weilte. Staat X 611 D wird der Meergott Glaukos geschildert, sein Körper ist durch die Wellen verunstaltet, von Muscheln, Seegras und Steinen überzogen. Dann wird auch einigemal auf die sagenhafte Urzeit Bezug genommen. So heißt es Protag. $320 \mathrm{C}$, es war einmal eine Zeit, wo es Götter gab, aber noch keine sterblichen Wesen, aus Erde und Feuer werden sie gebildet, die Geschichte ihrer Schöpfung wird ganz mythisch behandelt. Politikos $271 \mathrm{ff}$. wird das Leben unter der Regierung des Kronos geschildert. Friedliche, patriarchalische Zustände herrschten damals, weder Staat noch Familie gab es, aus Erde lebten alle wieder auf ohne Erinnerung an die Vergangenheit; reichliche Früchte hatten sie, die das Land von selbst hervorbrachte, nackt schliefen sie meist im Freien, denn ungünstige Witterung brauchten sie nicht zu fürchten, und weiche Lager gewährte ihnen das Gras, das massenhaft aus der Erde wuchs. Auch in den Gesetzen III 677 Aff. wird von diesem Urmenschentum gesprochen, besonders davon, wie es nach der Sintflut auf der Erde aussah. Nur Hirten hatten sich vor der Vernichtung auf die Berge gerettet, die Städte in der Ebene gingen zugrunde, und mit ihnen 
waren alle Errungenschaften der Kultur und Technik verloren. Wenige Menschen nur blieben übrig auf der ganzen Erde, einsam waren sie, und nur mit Furcht schritten sie von ihren Höhen hinab in die Ebenen $(678 \mathrm{C})$. Sie nährten sich von Milch und Fleisch und führten ein schlichtes, einförmiges Leben.

Bei all diesen selbständigen Naturschilderungen nun, seien sie mythisch gefärbt oder nicht, tritt zweifellos das starke Naturgefühl Platons zutage, aber es zeigt sich nicht so sehr in der reinen Anschauung der Natur als in ihrer praktischen Verwertung und in naturphilosophischer und phantasiemäßiger Konstruktion. Die Natur ist wesentlich auf den Menschen bezogen, wohl wird sie in ihrer Eigenart oft wissenschaftlich erforscht, und das subjektive Gefühl wendet sich ihr zu, aber es findet sich noch keine modern-sentimentale Hinneigung zu ihr, keine Stimmungsmalerei, wie sie erst durch die größere Distanz des Kulturmenschen von der Yatur möglich wird, der in die ihm fremden Objekte sein Sehnen und Wünschen hineinprojiziert und ihnen ein Eigenleben erteilt. Man darf bei dem Philosophen nicht lỵrische Ergüsse erwarten, aber die Griechen standen auch noch nicht in jenem lyrischen Gefühlsverhältnis zur Natur wie wir.

Den größten Anteil an den Naturschilderungen verschiedener Art haben Timäus, Phädrus, Kritias, Symposion, Staat, Gesetze, Phädon. Wir finden hier die Dialoge wieder, die auch bei den Gleichnissen und Metaphern usw. hervortraten. - Ganz interessant ist es zu sehen, welche Naturobjekte und Naturvorgänge überhaupt bei Platon hauptsächlich genannt werden. Hier stehen voran die Bezeichnungen, die sich auf die mannigfachen Gestalten und Ver-

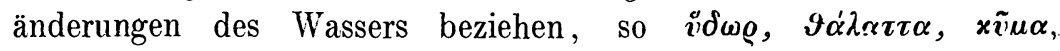

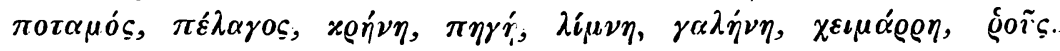
Es folgen die Bezeichnungen aus der Pflanzenwelt, besonders häufig

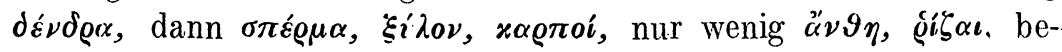
stimmte Namen werden nur vereinzelt genannt, wie $\delta \varrho \tilde{v} \varsigma, \pi \varepsilon \dot{v} * \eta$,

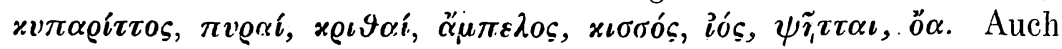
Himmel und Gestirne werden ziemlich häufig erwähnt, am meisten

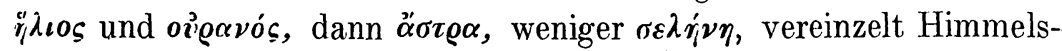

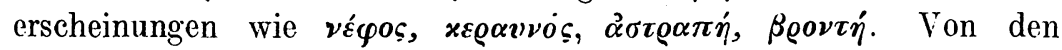
Ausdrücken, die sich auf die Erde beziehen, ist am meisten gebraucht

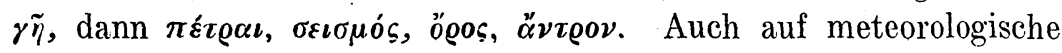
Vorgänge, Modifikationen der Jahreszeiten usw. wird mehrfach ein. 
gegangen: am häufigsten wohl werden genannt $\pi \nu \tilde{i} \gamma o \varsigma, \chi \varepsilon\llcorner\mu \omega \dot{\nu}$,

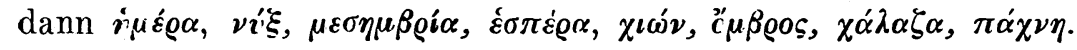
In bezug auf die Luft wird hauptsächlich von $\pi \nu \varepsilon \tilde{v} \mu \alpha$ geredet,

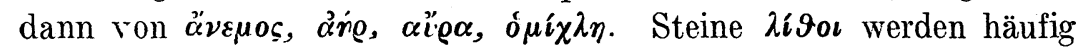
genannt, weniger bestimmte Mineralien. Auf das Feuer wird nur spärlich verwiesen. Größere, geschlossene Naturformationen wie Landṣchaften werden ziemlich selten erwähnt, am meisten noch ist

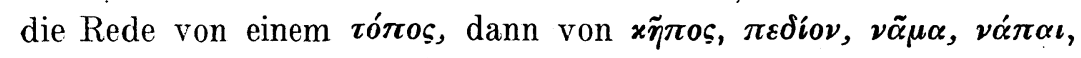
vereinzelt $\alpha^{\prime} \lambda \sigma o \varsigma, \vartheta \alpha^{\prime} \mu \nu \boldsymbol{s}, \dot{v} \psi \eta \lambda \dot{\alpha}, \lambda \varepsilon \imath \mu \boldsymbol{c}^{\prime} \nu$. Bestimmte Namen werden wenig verwendet, Ägypten mit dem Nil wird einigemal genannt, sonst nur nebenbei Sizilien, Ilissos, Knosos, Ilion, Euripos, abgesehen vom Kritias, der bei der Schilderung von Urattika mehrere geographische Namen bietet. - Auch aus diesen Zusammenstellungen ersieht man wohl, daß mit individueller Stimmung getränkte Naturbilder bei Platon kaum vorkommen. Das Naturgeschehen als solches interessiert den Philosophen, er sucht sein Wesen zu erforschen und nachzufühlen und die Natur geistigen Ideen zu unterwerfen.

Zum Schluß will ich noch kurz auf einige Äußerungen Platons hinweisen, die theoretisch das Verhältnis des Ich zur Natur in ästhetisch-gefühlsmäßiger Hinsicht behandeln. Mehrfach werden äußere Objekte des Schönen angeführt. Im Hippias mai. wird $288 \mathrm{D}$ eine

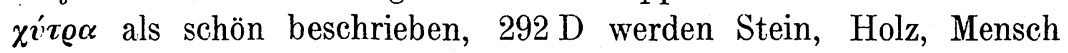
und Gott nebeneinander dargestellt, dann $295 \mathrm{C}$ heißt der menschliche Körper schön, ebenso werden Tiere erwähnt, in eigentümlicher . Auswahl (Pferd, Hahn, Wachtel). Phädon $78 \mathrm{D}$ sind Menschen, Pferde und Gewande Träger des Schönen; Phäd. $100 \mathrm{C}$ wird gefragt:

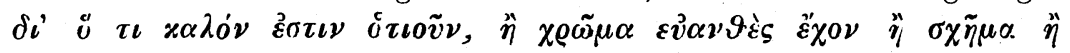

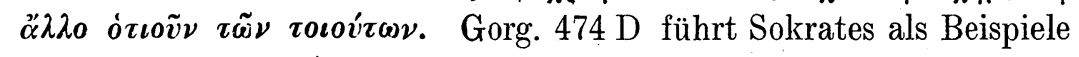

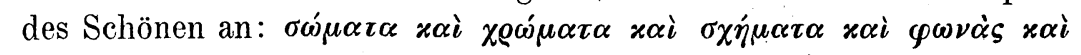
$\dot{\varepsilon} \pi \iota \tau \eta \delta \varepsilon \varepsilon^{\prime} \mu \iota \tau \alpha$. Das Schöne in Bewegung scheint Platon höher gestellt zu haben als das unbewegte (Tim. 19 B). Es gibt eine Stufenfolge in der Reihe des Schönen, die körperliche Schönheit steht niedriger als die geistige. Der Affe ist häßlich dem Menschen gegenüber, die schönste Vase häßlich mit einer Jungfrau verglichen, und alle menschliche Schönheit wird übertroffen von der göttlichen (Hipp. mai. 289 A, B). Staat III 401 A wird verschiedenen Künsten und Handwerken, dann aber auch der Natur der Körper und anderen

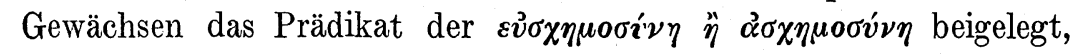


es wird eine Verwandtschaft zwischen Naturzuständen und Zuständen der Seele erkannt und der Natur neben dem ästhetischen auch ein ethischer Wert zugesprochen. Staat V 480 A heißt es, wir könnten Stimmen, schöne Farben und derartiges lieben, $\alpha \dot{v} \tau \dot{v}$ $\delta \dot{\varepsilon} \tau \dot{\boldsymbol{o}} x \alpha \lambda \dot{\boldsymbol{o}} \nu$

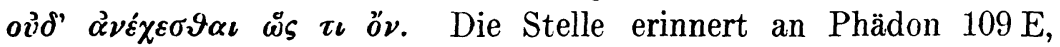
wo gesagt wird, wir würden den wahren Himmel und die wahre Erde erkennen, wenn unsere Natur die Anschauung ertragen könnte. Das Schöne in der Erscheinungswelt, wie wir es an den einzelnen Objeliten als Form oder Farbe usw. finden, hat nur einen relativen Wert, die absolute Schönheit, die Idee der Schönheit ist gänzlich verschieden davon (Sympos. $211 \mathrm{~A}$ ff.). Wenn nun aber der Philosoph über die unvollkommene Erscheinungswelt hinaus eine vollkommenere, harmonische, ideale Welt, die wahre Welt konstruiert, so kann doch der Mensch in seiner Bedingtheit durch die sinnliche Erfahrung diese niemals wahrnehmen. Die ästhetische Vorstellung also führt über die gegebene Wirklichkeit hinaus, daneben aber steht die Forderung der naturwissenschaftlichen Erkenntnis, die die Natur nach ihren Grundlagen und Gesetzen untersucht. Die Natur soll nicht anthropomorphisiert, sondern nach ihrer Eigenart und in ihrer Ganzheit betrachtet werden, wie das besonders deutlich Phädon $70 \mathrm{D}$ formuliert ist. Dort ist allerdings noch nicht von der späteren Naturphilosophie Platons die Rede, bei der Mathematik und Astronomie eine Hauptrolle spielen und die Vermenschlichung der Natur durchaus nicht so sehr vermieden ist.

Das Naturgefühl tritt uns bei Platon also in mannigfacher Form entgegen, was bedingt ist durch die mannigfachen Tendenzen seines Geistes. Kaum jedoch äußert es sich in subjektiv-lyrischer Weise, sondern es ist verbunden mit Reflexionen und Phantasievorstellungen, aber gerade hier können wir wohl einen Blick tun in die Seele Platons, in der künstlerische und wissenschaftliche Ideen sich verflechten zu einem Gebilde voll Erhabenheit und Schönheit. Sein Naturgefühl ist noch antik-klassisch, einzelne Spuren aber weisen schon auf das mehr romantische Gefühl des Hellenismus, von moderner Sentimentalität jedoch findet sich noch nichts. Platon wußte Kunst und Philosophie zur Harmonie zu bringen, allerdings nur, indem er die Kunst in seinem System zu sekundärer Bedeutung herabdrückte. 